\section{Cureus}

\title{
Intraperative Triggered Electromyography Recordings from the External Urethral Sphincter Muscles During Spine Surgeries
}

\author{
Faisal R. Jahangiri ${ }^{1}$, Rabehah A. Asdi ${ }^{2}$, Izabela Tarasiewicz ${ }^{3}$, Moutasem Azzubi ${ }^{4}$
}

1. Neurophysiology, Axis Neuromonitoring, Richardson, USA 2. Neurology, University of Texas at Dallas, Richardson, USA 3. Neurosurgery, University of Texas Health Science Center, San Antonio, USA 4. Neurosurgery, King Abdulaziz Medical City, Ministry of National Guard Health Affairs, Riyadh, SAU

$\square$ Corresponding author: Faisal R. Jahangiri, faisal.jahangiri@gmail.com Disclosures can be found in Additional Information at the end of the article

\section{Abstract}

Introduction: Bowel and bladder function are at risk during tumor resection and other surgeries of the conus, cauda equina, and nerve roots. This study demonstrates the ability to acquire triggered electromyography (t-EMG) from the external urethral sphincter (EUS) muscles by utilizing a urethral catheter with an electrode attached.

Methods: A retrospective analysis of neurophysiological monitoring data from two medical centers was performed. Seven intradural tumors and three tethered cord release surgeries that used urethral sphincter electrodes to record t-EMG were included in the analysis. The patients consisted of five females and five males with ages ranging from eight months to 67 years (median: 49 years). Our neuromonitoring paradigm included upper and lower extremity somatosensory evoked potentials (SSEPs) and transcranial electrical motor evoked potentials (TCeMEPs), as well as spontaneous and triggered electromyography (EMG) from the external anal sphincter (EAS), EUS muscles and lower extremity muscles bilaterally. A catheter with urethral electrodes attached was used for recording spontaneous electromyography (s-EMG), tEMG, and TCeMEPs from the skeletal muscle of the EUS. Train of four (TOF) was also recorded from the abductor hallucis muscle as well for monitoring the level of muscle relaxant.

Results: We were able to successfully record t-EMG responses from the EUS muscles in all patients (100\%). It is worthy to note that only one patient presented preoperatively with bladder incontinence, urgency, and frequency. Almost immediately in the postoperative phase, the patient's frequency and urgency improved, and the bladder function normalized within two weeks of having the tumor removed.

Received 06/03/2019 Review began 06/05/2019 Review ended 06/06/2019 Published 06/10/2019

\section{(c) Copyright 2019}

Jahangiri et al. This is an open access article distributed under the terms of the Creative Commons Attribution License CC-BY 3.0., which permits unrestricted use, distribution, and reproduction in any medium, provided the original author and source are credited.
Conclusions: In this small series, we were able to acquire t-EMG in $100 \%$ of patients when recorded from the EUS using a urethral catheter with electrodes built into it. T-EMGs can be attempted in surgeries that put the function of the pelvic floor at risk. More study is needed to establish better statistical methods, better modality efficacy, and a better understanding of intraoperative countermeasures that may be employed when an alert is encountered to prevent impending neurological sequelae.

Categories: Neurology, Neurosurgery, Orthopedics

Keywords: external urinary sphincters, urinary incontinence, electromyograph, emg, ionm, neuromonitoring, cauda equina tumors, tethered cord, sensory evoked potentials (ssep), motor evoked potentials (mep)

How to cite this article

Jahangiri F R, Asdi R A, Tarasiewicz I, et al. (June 10, 2019) Intraoperative Triggered Electromyography Recordings from the External Urethral Sphincter Muscles During Spine Surgeries. Cureus 11(6): e4867. DOI 10.7759/cureus.4867 


\section{Introduction}

The two urethral sphincters muscles are responsible for controlling micturition and for maintaining urinary continence. The smooth muscle internal urethral sphincter (IUS) is responsible for involuntary control and constriction of the internal urethral orifice. The striated external urethral sphincter (EUS) is responsible for a voluntary control and is part of the somatic nervous system [1]. The pudendal nerve (S2-S3-S4 nerve roots) controls the urethral sphincter. Any damage to the pudendal nerve or EUS may result in a lower urinary tract disorder. This would cause involuntary loss of urine, known as urinary incontinence. Women are more at risk of urinary incontinence because of the anatomy of the pelvic floor musculature and ligaments. During various surgeries, complications involving the urethral sphincter can lead to disorders including urinary incontinence, which can vary in men and women based on anatomical differences.

Bowel and bladder functions are at risk during various surgeries such as spinal surgeries, and tumor resections involving the conus, cauda equina and nerve roots. Multimodality intraoperative neurophysiological monitoring (IONM) is routinely performed during these procedures [2]. Bowel functions are usually recorded only from the external anal sphincter muscles (EAS) and monitored with electromyography (EMG), and transcranial electrical motor evoked potentials (TCeMEPs). Bladder function can now be monitored by TCeMEP from the EUS muscles [3]. Pudendal nerve somatosensory evoked potentials (PSEP) can also be used for monitoring the sensory roots of the pudendal nerve [3]. Somatosensory evoked potentials (SSEP) along with TCeMEP are used for monitoring of the spinal cord function. To monitor the bladder function directly triggered electromyography (t-EMG) can be utilized for intraoperative mapping of the pudendal nerve. This study demonstrates the ability to acquire t-EMG from the EUS muscles by utilizing a urethral catheter with an attached electrode.

T-EMG is an IONM technique that involves electrical stimulation of nerve roots using a handheld probe, and the measurement of compound muscle action potentials (CMAPs) from muscles innervated by these roots [4]. It is highly useful for surgeries involving the lower abdominal and pelvic areas which put bowel and bladder functions at high risk of neural damage [5]. Utilizing multimodality IONM allows surgeons to monitor the vital functions of the urethral sphincter throughout surgery and avoid causing damage to the surrounding nerves.

Surgeries that take place in the pelvic region have a high probability of causing damage to the bowel and bladder, leading to permanent and potentially fatal postoperative complications. In a study researching the incidence rate of postoperative urinary incontinence following spinal cord tumor surgeries without the use of IONM, it was found that over $83 \%$ of patients were left with urinary tract deficiencies [6]. Without IONM assistance and guiding the surgeon throughout the procedure, the likelihood of the patient experiencing bladder dysfunction is significantly increased. If the nerves innervating in the bladder are damaged, other lifethreatening complications such as incontinence or urinary retention may persist [7].

With the help of IONM, contemporary technology has allowed surgeons to intraoperatively monitor and test the receptiveness of the urinary sphincter using specialized electrodes to avoid causing lasting damage to the surrounding nerve roots. Triggered EMG recordings have very high specificity and are highly useful in aiding the preservation of autonomic nerves that innervate the bladder and sexual organs, as per the study by Delacroix et al. in 2010 [5].

\section{Materials And Methods}

We performed a retrospective analysis of neurophysiological monitoring data from 10 consecutive spinal surgeries. The data were collected from two medical centers in two countries. The patients consisted of five women and five men with ages ranging from eight 


\section{Cureus}

months to 67 years (median age: 49 years) (Figures 1-2). Seven patients were diagnosed with intradural tumors, and three patients with tethered cord syndrome. Our neuromonitoring protocol included bilateral upper and lower limbs SSEP, TCeMEPs, spontaneous EMG (s-EMG) and t-EMG from the EAS, EUS, and lower extremity muscles. For s-EMG, t-EMG, and TCeMEPs recording from the EUS muscles, a urinary catheter with an embedded bipolar urethral electrode (Signal Gear, Prosperity, SC, USA) was used [3]. A train of four (TOF) test was also performed from the abductor hallucis muscles in the foot.

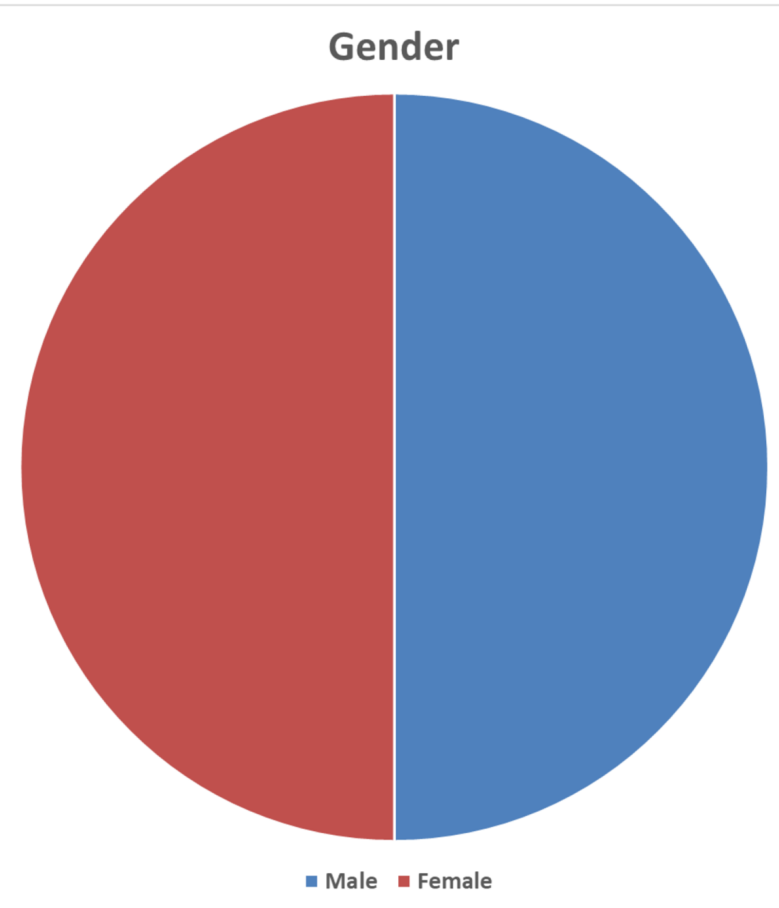

FIGURE 1: Gender distribution of the patients 


\section{Cureus}

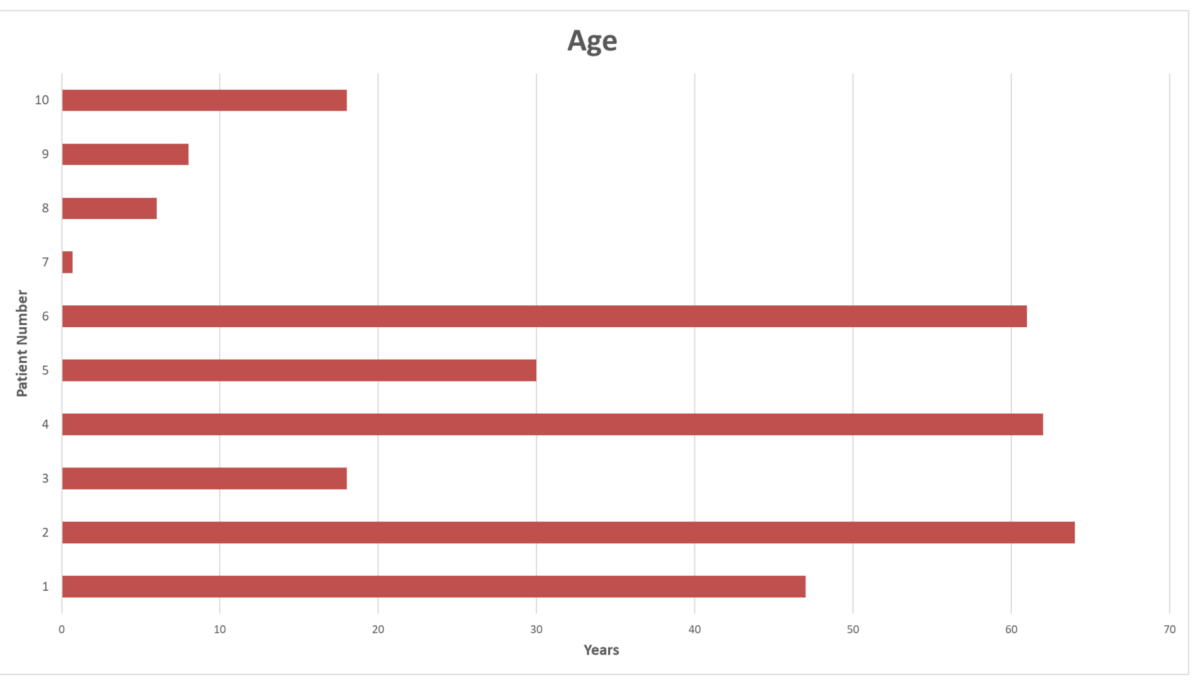

FIGURE 2: Age distribution of the patients

\section{Anesthesia protocol}

All the procedures were performed under general anesthesia utilizing total intravenous anesthesia (TIVA) with propofol and remifentanil infusions. In all patients, a short-acting neuromuscular blocking agent was used for intubation. A train of four (TOF) was recorded from the abductor hallucis muscle for monitoring the level of muscle relaxant throughout the surgical procedure [8].

\section{Intraoperative neurophysiological monitoring}

A multimodality IONM protocol, including SSEP, TCeMEP, EMG, and TOF, was utilized for all patients. Standard recommended parameters were used for SSEP stimulation and recordings [9]. EMG and TCMEP responses were recorded from adductor brevis, vastus medialis, tibialis anterior, gastrocnemius, abductor hallucis, EAS, and EUS muscles. For evaluation of the pelvic floor and pudendal nerve monitoring, EAS and EUS muscles were added to the neuromonitoring protocol. For TCeMEP stimulation, corkscrew electrodes were placed on the patient's scalp at C1, C2 and/or C3, C4 for. A train of 5-7 pulses was utilized (50-75 $\mu$ sec duration) with a stimulation intensity range of 120 to 380 volts.

A urinary catheter with attached urethral electrodes was used for recording t-EMG, s-EMG, and TCeMEPs from the skeletal muscle of the external urethral sphincters [3] (Figure 3). The size of urethral catheter electrodes was on the French scale (Fr.). The diameter of each unit is approximately $0.33 \mathrm{~mm}$ (e.g., a $12 \mathrm{Fr}$ catheter has a diameter of $4 \mathrm{~mm}$ ). Urethral catheter electrode sizes used for children: 8 Fr. or $10 \mathrm{Fr}$. and for adults $14 \mathrm{Fr}$. or $16 \mathrm{Fr}$. An appropriate size of the urethral catheter electrode was used per the patient's age, sex, urethral size, and weight. 


\section{Cureus}

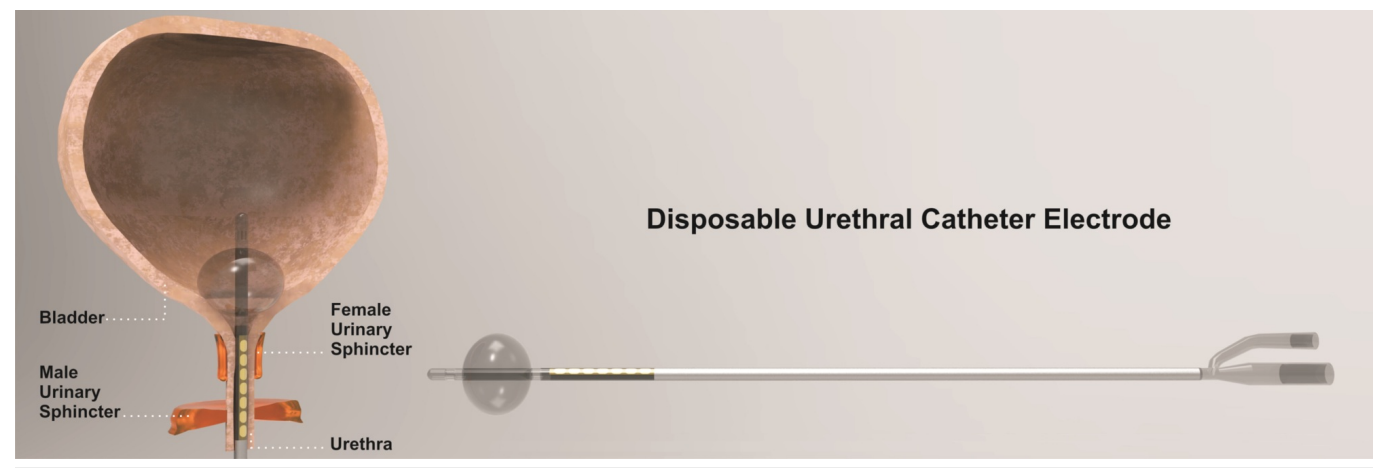

\section{FIGURE 3: Urethral catheter}

A disposable Foley catheter with an embedded bipolar urethral catheter electrode contacts for electromyography (EMG) and transcranial electrical motor evoked potential (TCeMEP) recordings (used with permission from Signal Gear, Prosperity, SC, USA).

A 32-channel Cadwell Cascade Pro (Cadwell Industries Inc, Kennewick, WA, USA) and Medtronic NIM-Eclipse (Medtronic, Inc., Minneapolis, MN, USA) were used for recorded multimodality IONM data during these surgical procedures.

\section{Results}

We were able to successfully record t-EMG responses in all patients (100\%) from the external urethral sphincter muscles (Figure 4). The morphology of the t-EMG responses from EUS was reliable and reproducible (Figures 5-6).

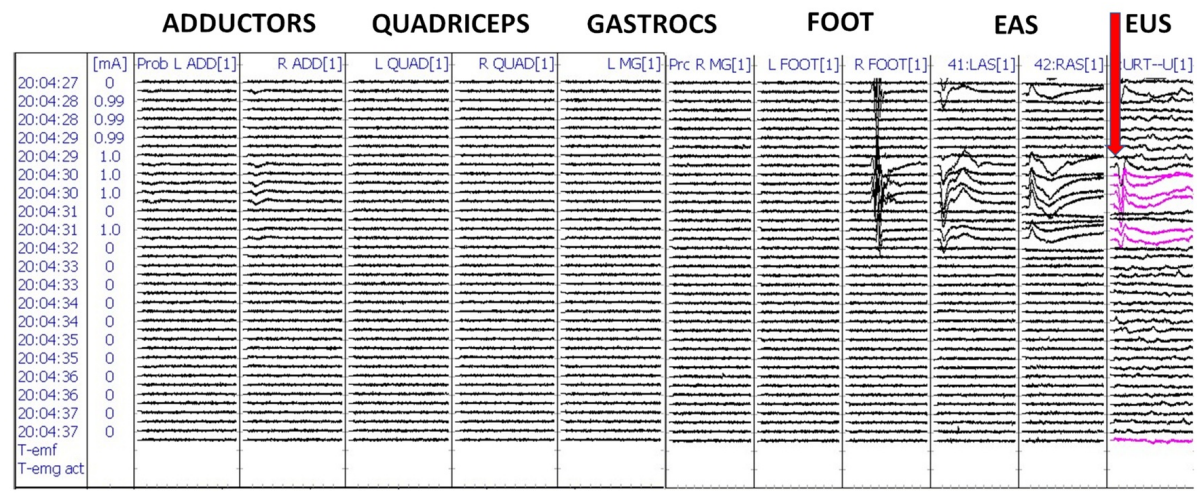

\section{FIGURE 4: Triggered electromyography (t-EMG) recordings} from the urethral sphincter electrodes in patient \#1

Red arrow: urethral electrode compound muscle action potentials (CMAP) responses from the external urethral sphincter (EUS). 


\section{Cureus}

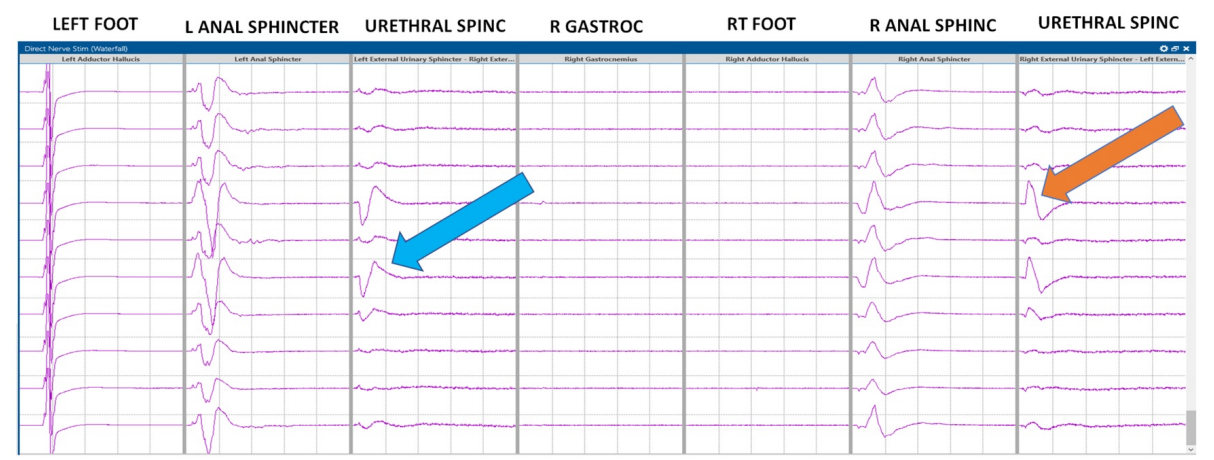

\section{FIGURE 5: Triggered electromyography (t-EMG) recordings from the urethral sphincter electrodes in patient \#9}

Blue and orange arrows: compound muscle action potentials (CMAP) responses from the external urethral sphincter (EUS).

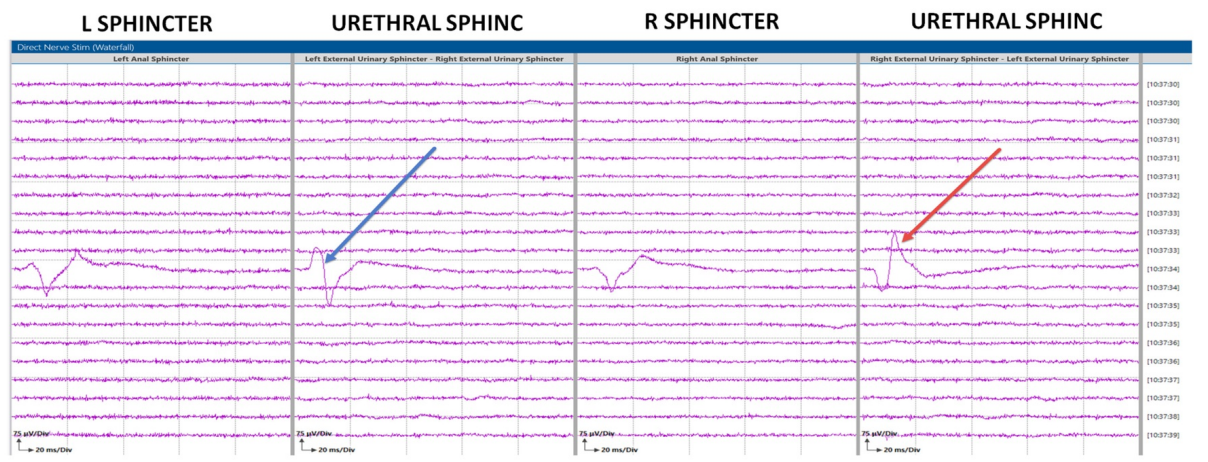

\section{FIGURE 6: Triggered electromyography (t-EMG) data}

External urethral electrode compound muscle action potential (CMAP) responses in patient \#2, indicated by the blue and red arrow.

Only one patient presented preoperatively with bladder incontinence, urgency, and frequency. The patient's frequency and urgency improved immediately in the postoperative phase, and the bladder function was normalized within two weeks of having the tumor removed. There were no false-negative or false-positive data in this study (Table 1). 


\section{Cureus}

\begin{tabular}{|c|c|c|c|c|c|c|}
\hline Patient & Age & Sex & Diagnosis & Surgery & Catheter Size & T-EMG \\
\hline 1 & $47 y$ & M & Cauda equina tumor & Laminectomy for tumor resection & $16 \mathrm{Fr}$ & $\mathrm{P}$ \\
\hline 2 & $64 y$ & $M$ & Conus tumor & Conus tumor resection & $16 \mathrm{Fr}$ & $\mathrm{P}$ \\
\hline 3 & $18 y$ & $\mathrm{~F}$ & Conus tumor & Conus tumor resection & $14 \mathrm{Fr}$ & $\mathrm{P}$ \\
\hline 4 & $62 y$ & $\mathrm{M}$ & Conus tumor & Conus tumor resection & $16 \mathrm{Fr}$ & $P$ \\
\hline 5 & $30 y$ & M & Cauda equina tumor & Conus tumor resection & $14 \mathrm{Fr}$ & $\mathrm{P}$ \\
\hline 6 & $61 y$ & M & Intradural tumor, L2-4 & Laminectomy for tumor resection & $16 \mathrm{Fr}$ & $\mathrm{P}$ \\
\hline 7 & $8 m$ & $\mathrm{~F}$ & Tethered cord & Tethered cord release & $8 \mathrm{Fr}$ & $\mathrm{P}$ \\
\hline 8 & $6 y$ & $\mathrm{~F}$ & Tethered cord & Tethered cord release & $10 \mathrm{Fr}$ & $\mathrm{P}$ \\
\hline 9 & $8 y$ & $\mathrm{~F}$ & Tethered cord & Tethered cord release & $10 \mathrm{Fr}$ & $\mathrm{P}$ \\
\hline 10 & $18 y$ & $\mathrm{~F}$ & Tethered cord & Tethered cord release & $16 \mathrm{Fr}$ & $\mathrm{P}$ \\
\hline
\end{tabular}

\section{TABLE 1: Triggered electromyography (t-EMG) responses recorded from the external urethral sphincter muscles (EUSM)}

$M=$ male,$F=$ female,$P=$ present,$F r=F r a n k$ size,$y=$ years,$m=$ months, $T$-EMG $=$ Triggered EMG.

\section{Discussion}

Incontinence is a poor outcome that surgeons want to avoid when the risk factor for incontinence is present [10-11]. Using t-EMG with UES muscle recordings provides a novel way the neurophysiologist and surgeon may be able to detect impending neurological sequelae, where other modalities that are currently used for neuromonitoring purposes have been focused on spinal cord monitoring [12].

For patients with an impaired bladder function due to an incomplete spinal cord injury (SCI) as a complication, we discovered that the pudendal SSEP's do not represent the autonomic function of the bladder, but rather somatic nerve fibers of the EUS showing that reproducible pudendal SSEP's are indicative of recovery of bladder function [13]. Another study that looked at EUS function on patients with SCI measured the level of recovered bladder function six months post trauma broken down into normal, impaired, and absent. Urodynamic examinations were done by using two micro transducer catheters to read urethral and rectal pressures as well as SSEP recordings from the extremities and gentiles [14]. They found that in acute tetraplegia patients, $27 \%$ recovered to normal bladder function, with $73 \%$ developing an upper motor neuron (UMN) lesion. The voluntary EUS function was normal in $27 \%$, impaired in $39 \%$, and absent in $34 \%$. Regarding paraplegic patients, only $10 \%$ recovered normal bladder function while $47 \%$ developed a UMN lesion, and 33\% developed a lower motor neuron (LMN) lesion while $8 \%$ had a combined lesion. The EUS was normal in the 10\%, impaired in $30 \%$ and absent in 60\% [14].

\section{Conclusions}


In this series, we were able to acquire t-EMG in $100 \%$ of patients when recorded from the EUS using a urethral catheter with electrodes built into it. T-EMGs can be attempted in surgeries that put the function of the pelvic floor at risk. More studies are needed to establish better statistical methods, better modality efficacy, and a better understanding of intraoperative countermeasures that may be employed when an alert is encountered to prevent impending neurological deficits. Currently, there are no studies published about neurophysiological monitoring of the urinary bladder or EUS function by utilizing t-EMG. This can be very helpful in surgeries involving the nerve roots supplying the EUS. This is the first study, to our knowledge, that shows a possibility for monitoring motor evoked potentials with EUS recordings in addition to the regularly monitored EAS.

\section{Additional Information \\ Disclosures}

Human subjects: Consent was obtained by all participants in this study. Animal subjects: All authors have confirmed that this study did not involve animal subjects or tissue. Conflicts of interest: In compliance with the ICMJE uniform disclosure form, all authors declare the following: Payment/services info: All authors have declared that no financial support was received from any organization for the submitted work. Financial relationships: All authors have declared that they have no financial relationships at present or within the previous three years with any organizations that might have an interest in the submitted work. Other relationships: All authors have declared that there are no other relationships or activities that could appear to have influenced the submitted work.

\section{Acknowledgements}

The authors would like to thank Brett Netherton, MS, CNIM, FASNM, FASET (Signal Gear LLC, Prosperity, SC USA) for providing his valuable support for this study.

\section{References}

1. Jung J, Ahn HK, Huh Y: Clinical and functional anatomy of the urethral sphincter . Int Neurourol J. 2012, 16:102. 10.5213/inj.2012.16.3.102

2. Siller S, Szelényi A, Herlitz L, Tonn JC, Zausinger S: Spinal cord hemangioblastomas: significance of intraoperative neurophysiological monitoring for resection and long-term outcome. J Neurosurg Spine. 2017, 26:411-545. 10.3171/2016.8.spine16595

3. Jahangiri FR, Silverstein JW, Trausch C, Al Eissa S, George ZM, Dewal H, Tarasiewicz I: Motor evoked potential recordings from the urethral sphincter muscles (USMEPs) during spine surgeries. Neurodiagn J. 2019, 59:34-44. 10.1080/21646821.2019.1572375

4. Mikula AL, Williams SK, Anderson PA: The use of intraoperative triggered electromyography to detect misplaced pedicle screws: a systematic review and meta-analysis. J Neurosurg Spine. 2016, 24:521-677. 10.3171/2015.6.spine141323

5. Delacroix S, Winters JC: Voiding dysfunction after pelvic colorectal surgery. Clin Colon Rectal Surg. 2010, 23:119-127. 10.1055/s-0030-1254299

6. Uchiyama T, Sakakibara R, Hattori T, Yamanishi T: Lower urinary tract dysfunctions in patients with spinal cord tumors. Neurourol Urodyn. 2003, 23:68-75. 10.1002/nau.10070

7. Geller EJ: Prevention and management of postoperative urinary retention after urogynecologic surgery. Int J Womens Health. 2014, 829. Accessed: May 10, 2019: 10.2147/ijwh.s55383

8. Ali HH, Utting JE, Gray C: Stimulus frequency in the detection of neuromuscular block in humans. Br J Anaesth. 1970, 42:967-78. 10.1093/bja/42.11.967

9. American Clinical Neurophysiological Society: Recommended standards for intraoperative monitoring of somatosensory evoked potentials. ACNS Guidelines and Consensus Statements. 2009, 1-10. Accessed: May 15, 2019: https://www.acns.org/pdf/guidelines/Guideline-11B.pdf.

10. Roblick UJ, Bader FG, Jungbluth T, Laubert T, Bruch HP: How to do it-laparoscopic resection 


\section{Cureus}

rectopexy. Langenbecks Arch Surg. 2011, 396:851-5. 10.1007/s00423-011-0796-5

11. Byrne CM, Smith SR, Solomon MJ, Young JM, Eyers AA, Young CJ: Long-term functional outcomes after laparoscopic and open rectopexy for the treatment of rectal prolapse. Dis Colon Rectum. 2008, 51:1597-604. 10.1007/s10350-008-9365-6

12. Kauff DW, Koch KP, Somerlik KH, Hoffmann KP, Lang H, Kneist W: Evaluation of twodimensional intraoperative neuromonitoring for predicting urinary and anorectal function after rectal cancer surgery. Int J Colorectal Dis. 2013, 28:659-664. 10.1007/s00384-013-1662-4

13. Curt A, Dietz V: Electrophysiological recordings in patients with spinal cord injury: significance for predicting outcome. Spinal Cord. 1999, 37:157-165. 10.1038/sj.sc.3100809

14. Curt A, Rodic B, Schurch B, Dietz V: Recovery of bladder function in patients with acute spinal cord injury: significance of ASIA scores and somatosensory evoked potentials. Spinal Cord. 1997, 35:368-373. 10.1038/sj.sc.3100423 